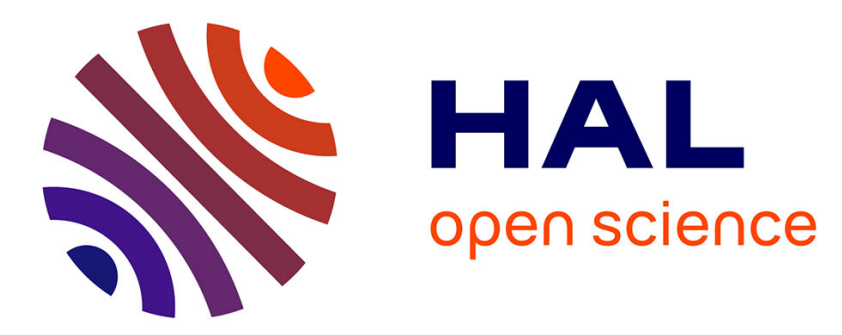

\title{
Butterfly species differing in mobility show different structures of dispersal-related syndromes in the same fragmented landscape
}

S Ducatez, A Humeau, M Congretel, Helene H. Freville, Michel Baguette

\section{- To cite this version:}

S Ducatez, A Humeau, M Congretel, Helene H. Freville, Michel Baguette. Butterfly species differing in mobility show different structures of dispersal-related syndromes in the same fragmented landscape. Ecography, 2013, 37 (4), pp.378-389. 10.1111/j.1600-0587.2013.00365.x . hal-01310325

\section{HAL Id: hal-01310325 \\ https://hal.science/hal-01310325}

Submitted on 14 Oct 2020

HAL is a multi-disciplinary open access archive for the deposit and dissemination of scientific research documents, whether they are published or not. The documents may come from teaching and research institutions in France or abroad, or from public or private research centers.
L'archive ouverte pluridisciplinaire HAL, est destinée au dépôt et à la diffusion de documents scientifiques de niveau recherche, publiés ou non, émanant des établissements d'enseignement et de recherche français ou étrangers, des laboratoires publics ou privés. 


\title{
Butterfly species differing in mobility show different structures of dispersal-related syndromes in the same fragmented landscape
}

\author{
S. Ducatez, A. Humeau, M. Congretel, H. Fréville and M. Baguette
}

S. Ducatez (simon.ducatez@mail.mcgill.ca), A. Humeau, M. Congretel and H. Fréville, Muséum National d'Histoire Naturelle, Dépt Ecologie et Gestion de la Biodiversité, 57 rue Cuvier, FR-75005 Paris cedex 5, France. SD also at: Dept of Biology, McGill Univ., 1205 Avenue Docteur Penfield, Montréal, QC H3A 1B1, Canada. AH also at: Inst. de Recherche sur la Biologie de l'Insecte, CNRS UMR 7261, Univ. François Rabelais, Avenue Monge Parc Gradmont, FR-37200 Tours, France. HF also at: CEFE CNRS UMR 5175 , 1919 route de Mende, FR-34293 Montpellier cedex 5, France. - M. Baguette, Muséum National d'Histoire Naturelle, UMR 7205 OSEB, 57 rue Cuvier, FR-75005 Paris cedex 5, France, and Station d'Ecologie Expérimentale du CNRS à Moulis, CNRS USR 2936, Moulis, FR-09200 Saint-Girons, France.

\begin{abstract}
Mobility varies strongly between and within species, reflecting different dispersal strategies. Within species, such differences can imply suites of traits associated in syndromes. Different syndrome structures have been found within species among populations differing in the selective pressures they are exposed to. Similarly, we expect species differing in mobility to show different syndrome structures in response to similar selective pressures such as landscape fragmentation. Using butterflies originating from the same fragmented landscape, we investigated the differences in mobility syndrome between four common butterflies (Pyronia tithonus, Pararge aegeria Maniola jurtina, Pieris rapae) known to differ in their mobility. We expected individuals from the less mobile species to display a resident strategy because of high dispersal cost in this fragmented landscape, and individuals from the more mobile species to display a larger range of movement strategies. Moreover, as syndromes can only be detected whenever individuals differ in their dispersal strategies, we expected mobility syndromes to be observable only in populations where dispersal polymorphism is maintained. We thus expected stronger correlations between mobility-related traits in more mobile species. Using three mobility tests in controlled conditions designed to measure different components of mobility, we showed that mobility-related traits were indeed correlated only in the most mobile species. The absence of correlation in the less mobile species may be explained by a low variation in movement strategies, dispersal being counter-selected.
\end{abstract}

Dispersal evolution is driven by the balance between fitness costs and benefits associated with dispersal decision. This balance differs according to both external conditions and phenotypes, so that dispersal is both condition and phenotype dependent (Clobert et al. 2012). Phenotypic differences between dispersers and residents are widespread across taxa, and such differences are supposed to reflect a specialization in dispersal-related life-history strategy (Clobert et al. 2009, 2012). Dispersal strategies (e.g. resident and disperser strategies) have been shown to involve suites of correlated traits called syndromes (Clobert et al. 2009, Cote et al. 2010). Associations between dispersal and morphological, physiological and behavioral traits are indeed expected to increase dispersal success by decreasing the costs associated with dispersal (Clobert et al. 2009, Cote et al. 2010, Bonte et al. 2012). For instance, in natural populations of great tits Parus major, natal dispersal distance has been found to be correlated with exploratory behavior, immigrants being faster explorers than philopatric individuals (Dingemanse et al. 2003).
Differences in dispersal strategies have been shown to be heritable in a variety of species, including birds (Hansson et al. 2003, Doligez et al. 2009, Charmantier et al. 2011) and plants (Cheptou et al. 2008). In butterflies, genotype-dependent dispersal has been documented in Melitaea cinxia, where genetic variation at the metabolic enzyme phosphoglucose isomerase (Pgi) locus has been shown to correlate with variation in individual dispersal propensity in natural populations (Haag et al. 2005, Niitepold et al. 2009, Wheat 2012). This locus is known to influence physiological characteristics (e.g. flight metabolic rate) that likely affect flight, and thus dispersal ability. Overall, these results suggest that evolution by natural selection is likely to affect the maintenance of different dispersal strategies (or dispersal polymorphism) in natural populations of many species. Within a species, the maintenance of dispersal polymorphism by natural selection may largely vary according to environmental conditions. Indeed, dispersal rate is known to vary according to environmental conditions (Clobert et al. 2012). For instance, the level of landscape 
fragmentation has been shown to affect the proportion of dispersing individuals in many species (Cheptou et al. 2008, Baguette et al. 2012, Clobert et al. 2012, Van Dyck and Baguette 2012). This effect may reflect a phenotypic response to landscape fragmentation, individuals directly responding to the environment when deciding to disperse, or a genetic response by microevolution, both affecting the maintenance of dispersal polymorphism (i.e. differently specialized phenotypes) in a given environment.

In this framework, detecting dispersal syndromes, i.e. suites of correlated dispersal-related traits is expected only when different dispersal strategies are maintained within populations. Although not focused on dispersal, some intraspecific studies have indeed shown variations in behavioral syndromes depending on environmental conditions (Dingemanse et al. 2007, 2009, 2012a, b). For instance, in the three-spined stickleback Gasterosteus aculeatus, Dingemanse et al. (2007) have found a behavioral syndrome associating aggressiveness, activity and exploratory behaviors only in populations from large ponds where predators were present, suggesting that different behavioral strategies are only maintained in the presence of predators. Similar patterns are expected with regards to dispersal syndromes whenever selective pressures arising from variations in environmental conditions such as landscape fragmentation affect the maintenance of dispersal polymorphism.

The balance between the costs and benefits of dispersal may also vary among species inhabiting the same landscape (Bonte et al. 2012, Van Dyck and Baguette 2012), according to species differences in traits affecting dispersal costs such as mobility, social structure or habitat specialization. Similar environmental conditions may then lead to between-species differences in selective pressures acting on the maintenance of dispersal polymorphism. Such differences can be detected by testing for differences in syndrome structure across species. For instance, at a high level of landscape fragmentation, poorly mobile species may entail strong dispersal costs, so that natural selection would select against dispersing phenotypes, and only resident phenotypes would remain in the population (Fig. 1). In this case, no dispersal syndrome would be detected. In contrast, in highly mobile species, lower dispersal costs may allow the maintenance of disperser and resident strategies at high levels of landscape fragmentation, dispersal syndromes being then detectable (Fig. 1). More mobile species are thus expected to maintain a higher variation in dispersal strategies than less mobile species at high levels of landscape fragmentation (Fig. 1). However, to our best knowledge, no previous study has directly investigated inter-specific differences in dispersal syndromes for species inhabiting the same landscape, likely because of the difficulty to make reliable comparisons.

Mobility, defined here as the propensity to make displacements, is the net result of the interaction between morphology, physiological performances and movement decisions (Ducatez et al. 2012). These different components are likely to be interrelated in a common syndrome, provided that they experience similar selective pressures acting on mobility. Moreover, mobility is expected to strongly influence dispersal movements (Ronce 2007). We may thus expect to find a strong association between mobility syndromes and dispersal, such a mobility syndrome being likely to reflect different mobility-dependent strategies that are related to dispersal. In this context, butterflies are particularly well-suited model species as mobility (i.e. the propensity to make displacements) and dispersal (i.e. the actual displacement from site of birth to site of reproduction, or between successive reproduction sites) are known to strongly vary both between and within species in this taxonomic group (Stevens et al. 2010a, b). Accordingly, a mobility syndrome implying different mobility-related traits has been found in the butterfly Pieris brassicae (Ducatez et al. 2012). Moreover, one of the traits involved in the syndrome (flight endurance under stressful conditions) has been shown to correlate with dispersal, butterflies flying a longer time in stressful conditions being also more likely to disperse in experimental metapopulations (D. Legrand pers. comm., Ducatez 2011). This mobility syndrome thus directly affects inter-individual variation in dispersal in this species.

In this study, we tested differences in dispersal-related mobility syndromes among four butterfly species Maniola jurtina, Pararge aegeria, Pieris rapae and Pyronia tithonus inhabiting the same fragmented landscape, by measuring a set of mobility traits on individuals collected in an urban park situated in a highly urbanized area where they reproduce. Those four species have already been shown to differ in their mobility in the wild (Thomas 1984, Pollard and Yates 1993, Dennis and Shreeve 1997, Cook et al. 2001). Differences in dispersal syndrome among species are likely to be found at intermediate-high fragmentation of landscape because dispersal costs are high and only highly mobile individuals can overcome the costs of dispersal. We expected more mobile species to have stronger withinindividual consistency in mobility-related traits across time and stronger correlations between traits than less mobile species. To test those predictions, we first measured three mobility-related traits in each species in controlled conditions, using three tests specifically designed to measure different components of mobility (Ducatez et al. 2012), including movement capacity and movement decision to assess the validity of the ranking of inter-specific differences in mobility observed in other studies (Thomas 1984, Pollard and Yates 1993, Dennis and Shreeve 1997, Cook et al. 2001). We also measured butterflies' heating rate as a physiological trait potentially affecting butterflies' mobility (Ducatez 2011, Ducatez et al. 2013). We then tested whether species differed in the repeatability across time of mobility-related traits and tested whether the correlations between traits depended on species mobility ranks.

\section{Material and methods}

\section{Study system}

The four species are abundant in France and Europe (Lafranchis 2004) and have a wide distribution, from Maghreb to Russia or even Japan. They are generalist species, caterpillars feeding on a wide range of Poaceae species (M. jurtina, P. aegeria and P. tithonus) and Brassicaceae species (P. rapae). They however differ in their habitats (Lafranchis 2004); M. jurtina and P. tithonus are grassland species, 

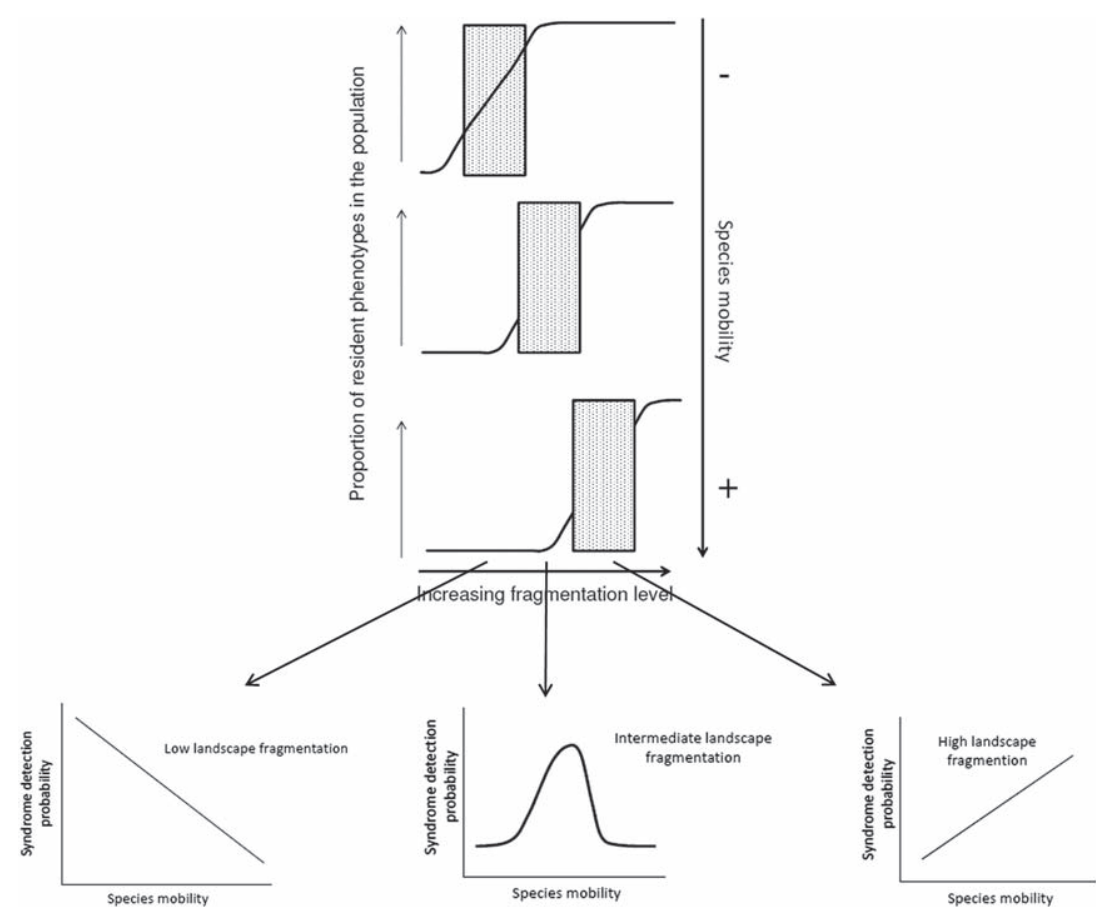

Figure 1. Hypothetical relationships between the expected dispersal polymorphism maintenance (maintenance of resident and disperser strategies) and dispersal costs associated with landscape fragmentation (3 upper graphs), and the resulting expected levels of fragmentation where mobility syndromes is detectable (3 bottom graphs). Note that to make the figure clearer and focus on the effects we aimed at testing (i.e. the evolution of dispersal polymorphism with species mobility), we here consider extreme cases, with no polymorphism at low and high fragmentation levels, and no evolution of species mobility with landscape fragmentation (i.e. species could only respond to landscape fragmentation by altering the proportion of dispersers and residents, but could not evolve higher mobility). Species mobility is considered here as a fundamental factor affecting dispersal costs (less mobile species entailing higher costs for a given fragmentation level), the interaction between landscape fragmentation and mobility thus affecting the maintenance of inter-individual variation in dispersal strategies and thus variation in dispersal-related phenotypes. Polymorphism in dispersal-related traits is expected for intermediate dispersal costs. Upper panel: grey areas represent landscape fragmentation levels where dispersal polymorphism is expected, making mobility syndromes detectable. At low fragmentation levels (left of the 3 graphs), we expect most individuals to have a disperser phenotype, as dispersal costs are very low. Poorly mobile species are however likely to entail comparatively more dispersal costs than more mobile species, and thus to display some polymorphism in dispersal strategy. Increasing fragmentation will increase the costs of dispersal, and so more quickly for poorly mobile species. Thus, at intermediate fragmentation levels (middle of the 3 graphs), the maintenance of polymorphism is expected for species with intermediate mobility (and intermediate dispersal costs, middle graph), whereas we expect to find mainly residents in poorly mobile species (and high dispersal costs, upper graph), and mainly dispersers in highly mobile species (and low dispersal costs, graph at the bottom). Finally, at high fragmentation level (right part of the three graphs), we expect dispersal polymorphism only in highly mobile species (graph at the bottom). The fragmentation level investigated in this study is expected to be intermediate to high (grey area on the figure). Bottom panel: for a given level of landscape fragmentation, mobility syndromes are detectable only when dispersal polymorphism exists, so in the grey area of the upper panel. The bottom panel details the relationship between the probability to observe dispersal syndrome and species mobility at three different fragmentation levels. Arrows indicate the correspondence between fragmentation levels on the upper and bottom panels. At low fragmentation level, the probability of detecting mobility syndromes will thus decrease with species mobility (poorly mobile species may show some polymorphism, whereas intermediate and highly mobile species will mainly be composed of dispersers). At intermediate fragmentation level, polymorphism in dispersal strategy is expected for species with intermediate mobility, so that a mobility syndrome can be detected for these species, but not for poorly or highly mobile species. Finally, at high fragmentation levels, polymorphism in dispersal strategy and the detection of a mobility syndrome are expected only for highly mobile species. This last graph corresponds to this study's situation.

whereas $P$. aegeria is a woodland species and $P$. rapae inhabits any type of open habitat. M. jurtina, P. tithonus and P. aegeria are in the same Nymphalidae family whereas $P$. rapae belongs to the Pieridae family. Although no phylogenetic tree with branch lengths exists for European butterflies, information on the four studied species' phylogenetic position is available in a consensual phylogenetic tree without branch length provided by Cizek et al. (2006). According to this tree, $M$. jurtina and $P$. tithonus are the most closely related species among the four considered. These two species and $P$. aegeria are then grouped in a clade, $P$. rapae being the most distant from the three other species. We collected butterflies with a net in the same urban park located in Brunoy (Essonne, France; lat: $48^{\circ} 41^{\prime} 40.50^{\prime \prime} \mathrm{N}$, long: $2^{\circ} 29^{\prime} 47.71^{\prime \prime} \mathrm{E}$ ) every day between 8 July 2010 and 24 August 2010 between $10 \mathrm{am}$ and $3 \mathrm{pm}$, weather permitting. We captured a total of 358 individuals (Fig. 2) that we kept in cages of $20 \times 20 \times 20 \mathrm{~cm}$ with artificial flowers containing a solution of $10 \%$ sugar in water. We then measured mobility traits and heating rate for each individual on their capture day. Mean dry body mass, estimated from the butterflies used in this study, was as follows (mean \pm SE): P. tithonus: $11.28 \pm 0.78 \mathrm{~g}$, M. jurtina: $19.84 \pm 1.34 \mathrm{~g}, P$. aegeria: $10.60 \pm 0.24 \mathrm{~g}$, P. rapae: $14.24 \pm 0.30 \mathrm{~g}$. 

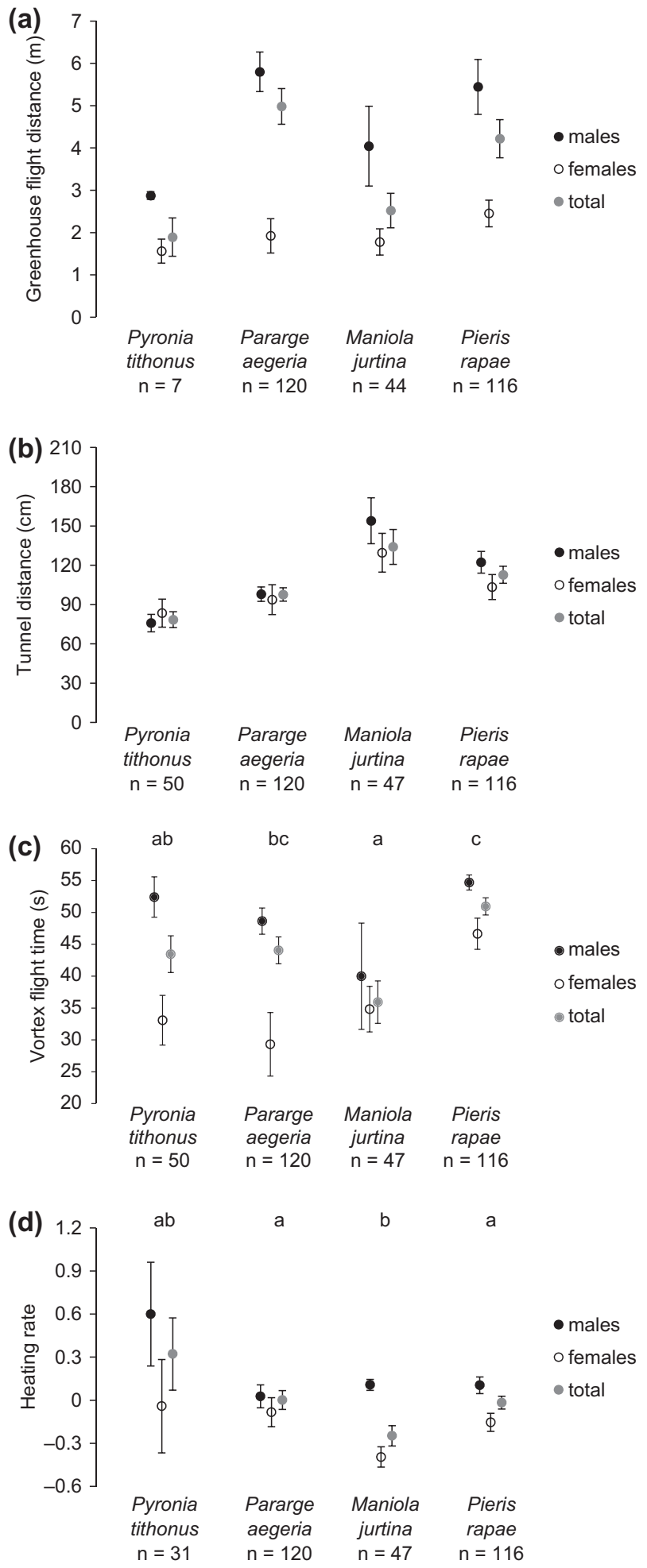

Figure 2. Mean mobility scores of four butterfly species measured for four mobility-related traits. Error bars are standard errors. Heating rate was corrected by thorax temperature differences at the beginning of the measure (see text for details). Species were ordered according to their mobility rank (see text), from the less mobile to the most mobile, $P$. aegeria and $M$. jurtina sharing the same rank. The significance of between-species differences is presented for the two tests where the species effect was significant (i.e. vortex and heating rate, see text for details).

\section{Measurements of mobility-related traits}

We characterized each individual of each species by measuring in controlled conditions four traits that have been shown to reflect different behavioral and physiological components involved in mobility (Ducatez 2011, Ducatez et al. 2012).

\section{Greenhouse test}

The greenhouse test was used to characterize in seminatural conditions both the physiological capacity of butterflies to move in the greenhouse and the exploration tendency (behavioral aspect) (Ducatez et al. 2012). We supposed all individuals were physiologically able to cross the $48 \mathrm{~m}$ long greenhouse. The four studied species are indeed known to travel over several hundreds of meters to several kilometers. However, some may perform better because of the variation in particular physiological traits such as thorax muscular mass or flight metabolic rate that are likely to influence the exploration decision, or because of differences in exploration tendency. Butterflies were individually released at $1-\mathrm{m}$ height at the extremity of a $48 \mathrm{~m}$ long $\times 8 \mathrm{~m}$ wide $\times 4 \mathrm{~m}$ high greenhouse covered with a mesh and located in a field at the Museum National d'Histoire Naturelle in Brunoy, France. We measured their flight distance (distance to the first landing in meters) over four trials. For each individual, the time elapsed between the first and the fourth trial was between 30 and $60 \mathrm{~min}$, which allowed repeatability estimates (see below). Flight distance was estimated by dividing the greenhouse into 72 squares (the greenhouse was divided into 3 squares widthwise and 24 lengthwise), and recording the butterfly landing square. We then calculated the Euclidean distance between the release point and the square center. Butterflies that flew the longer distances in the greenhouse were considered good explorers and performers, whereas butterflies that flew short distances were considered bad explorers and bad performers. This test was only performed on sunny days with ambient temperatures ranging from 25 to $30^{\circ} \mathrm{C}$, i.e. temperature conditions ensuring flight activity. As a result, only 289 individuals among the 358 were tested in the greenhouse.

\section{Tunnel test}

The aim of the tunnel test was to measure movement willingness under stressful and unfamiliar conditions (Ducatez et al. 2012). In a dark room held at a constant temperature of $24 \pm 1^{\circ} \mathrm{C}$, butterflies were individually released at the entrance of a 3-m-long opaque pipe of $80 \mathrm{~cm}$ diameter with a weak light source at its end. Each individual was released in the air, at a height of $40 \mathrm{~cm}$, to avoid any take-off effort so that test scores mainly depended on a behavioral choice rather than a physiological capacity. This test was specifically designed to test for the individual bold/shy tendency. The novelty of this experimental situation, in addition to the small diameter and the darkness of the tunnel, was particularly challenging to the butterflies. We thus expected butterflies with a bold temperament to go far in the tunnel, and butterflies with a shy temperament to stay at its entry. We observed strong behavioral differences among individuals: the distance individuals moved in the 
tunnel ranged from 0 to $300 \mathrm{~cm}$ (i.e. the tunnel length). We performed five trials carried out within less than an hour for each individual, to test for behavioral repeatability (see below). A test was considered as finished as soon as the butterfly stopped moving during more than $5 \mathrm{~s}$. For each trial, we recorded the travelled distance (tunnel distance), shy individuals being expected to travel shorter distances. Shy individuals could also be expected to cross the tunnel faster, in which case tunnel crossing could be considered as an escape behavior rather than result from a higher boldness. We would then expect individuals crossing the tunnel to be overall faster than those which stopped inside the tunnel. We however found that, within the 4 tested species, the relationships between tunnel distance and time (i.e. the time needed to travel the tunnel distance; this variable is not further used in this study) were strong and linear $\left(\mathrm{R}^{2}=0.24\right.$ to 0.60 according to species), and individuals crossing the tunnel did not show any tendency to be faster (data not shown). The tunnel distance variable thus provides a relevant measure of movement willingness under stressful and unfamiliar conditions, which can be interpreted as an estimate of boldness.

\section{Vortex test}

The vortex test was designed to detect individual differences in flight endurance under stressful conditions (Ducatez et al. 2012). Each butterfly was individually introduced within a $25 \times 10 \times 10 \mathrm{~cm}$ plastic chamber perforated at its base and fixed to a rapid agitator. The temperature of the chamber was kept constant at $25 \pm 1^{\circ} \mathrm{C}$. We then turned the agitator on for one minute, resulting in strong shaking of the chamber which impeded the butterfly to perch on the chamber's wall. Each butterfly could either fly in a reduced volume or lay uncomfortably at the bottom of the chamber in a reduced area strongly shaken, both behaviors likely to be resource demanding due to stressful conditions. We measured the cumulative time each individual spent flying during the test (hereafter referred to as flight time), higher values reflecting better movement abilities. The duration of this test was chosen to limit any negative effects on individual wings, wing morphology being measured the day after the tests (Supplementary material Appendix 1). For this reason, we did not measure the repeatability of flight time. This test has been found to positively correlate with dispersal in experimental metapopulations in P. brassicae, dispersers having significantly higher vortex scores than residents (D. Legrand pers. comm., Ducatez 2011).

\section{Heating rate measurement}

We assessed the heating rate of individuals, a variable that has already been shown to be positively correlated with mobility in P. brassicae butterflies (Ducatez 2011). To do so, we performed a warming experiment in the laboratory. Each butterfly was first cooled in a refrigerator $\left(4^{\circ} \mathrm{C}\right)$ for $10 \mathrm{~min}$, and then warmed up at $27^{\circ} \mathrm{C}$ during $180 \mathrm{~s}$ under a $300 \mathrm{~W}$ Ultra vitalux solar lamp placed at a distance of $72 \mathrm{~cm}$, while its thorax temperature was measured continuously with an infrared thermometer (emissivity $\varepsilon=0.95$, one data point $\mathrm{s}^{-1}$ until $180 \mathrm{~s}$ ). The heating rate was expressed as the slope of the thorax temperature versus time (log transformed) curve (Van Dyck and Matthysen 1998, Ducatez et al. 2013); in our data, the corresponding linear regression very well described the increase in thorax temperature with time $\left(r^{2}=0.99 \pm 0.00\right)$. Note that, as individuals' temperature at the end of the cooling period differed as a possible consequence of differences in body mass, the heating rate was regressed against the individual temperature at the beginning of the measure. We used the residuals of this regression to have a standardized estimate of heating rate (hereafter called heating rate). Butterflies that warmed up fast were characterized by high heating rate values.

\section{Experimental protocol}

Each individual experienced the same temporal sequence of mobility tests. Butterflies first performed the greenhouse test, followed by the tunnel test. They were then assessed for their heating rate and they finally performed the vortex test. For each individual, less than five hours elapsed between the first and last tests. One day after their capture, we photographed the butterflies for morphological measurements (Supplementary material Appendix 1) and estimated an age index based on the wear of the wings, varying from 1 [fresh] to 4 [extensive wing wear] (see capture mark recapture experiments on butterflies: Baguette and Nève 1994). The greenhouse test and the warming experiment for the heating rate measurement were the most time consuming tests. For logistical reasons, and to maintain a fixed duration between the first test and the last one, we did not manage to test all individuals for those two tests: out of 358 collected butterflies, 289 performed the greenhouse test and 302 were assessed for their heating rate. Although we initially aimed at testing $P$. tithonus in the greenhouse, the small size of this species made it highly challenging to observe released individuals in the greenhouse and to find them on the ground once they landed, so that we were not able to take four accurate measurements of greenhouse distance for most $P$. tithonus captured at the beginning of the testing period. We thus finally decided not to test this species anymore in the greenhouse, and only present results for the 7 P. tithonus individuals which were initially tested in the greenhouse. The remaining individuals were only tested in the tunnel and vortex tests. The number of tested individuals for each species and each test is given in Fig. 2.

\section{Data analysis}

We first ranked species according to their expected mobility (Dennis and Schreeve 1997, Cook et al. 2001). Pieris rapae is considered as having a nomadic strategy of space use (sensu Mueller and Fagan 2008), living in open populations whereas the three other species are supposed to live in closed populations (Thomas 1984, Pollard and Yates 1993). We thus attributed the highest mobility rank to Pieris rapae as compared to the three other species. In addition, according to migration indices measured through two different methods (Dennis and Schreeve 1997, Cook et al. 2001), P. tithonus appears as having the lowest mobility, whereas $P$. aegeria and $M$. jurtina display intermediate levels of mobility. These migration indices focus on the distances species have been recorded covering, and take into account different components of movement including the occurrence of ex-habitat vagrants, recorded range expansions, at sea records and records of mass-movements (see Dennis 
and Schreeve 1997, Cook et al. 2001 for details). Pyronia tithonus was thus attributed a mobility rank of $1, P$. aegeria and $M$. jurtina a rank of 2 and $P$. rapae a rank of 3 .

\section{Among-species differences in mobility-related traits}

We first tested whether differences in mobility-related traits depended on species mobility rank by using linear models with mobility score at each of the tests as a response variable and mobility rank, age and sex, as well as their second order interaction as fixed explanatory variables. Note for the tunnel test and the greenhouse test that each individual performed respectively five and four times, we first calculated a mean score for each individual. Second order interactions and age were never significant, and we thus excluded them from the models. Considering the greenhouse test, we also added the time of the day and the date of the test as fixed factors to correct for potential climatic differences experienced by individuals. Those latter factors were never significant and were thus excluded from the models. Tunnel distance and greenhouse flight distance were $\log$-transformed to achieve normality and homoscedasticity assumptions. Because flight time in the vortex was not normally distributed (even after transformation), we used non-parametric test to assess differences in flight time between species. We first tested whether flight time differed across species using a Kruskal-Wallis test. Then, we compared flight time between each pair of species using Wilcoxon rank order tests, and used Bonferroni corrections to account for multiple comparisons. We finally compared flight time between males and females within each species, after having corrected for multiple tests.

\section{Individual repeatability of mobility score across time}

For the tunnel test and the greenhouse test, we estimated individual repeatability for each test and tested whether it varied with species mobility rank. Individual repeatability was calculated as the ratio of the between-individual variance over the sum of the between- and within-individual variance (Lynch and Walsh 1998). Variance components were estimated from linear mixed models (LMM) with the test score as response variable, the trial rank as a fixed effect and the individual as a random effect. Note that the trial rank effect was never significant and was thus excluded from the models. Confidence intervals and tests for significance were computed following Nakagawa and Schielzeth (2010). We thus used the $\mathrm{R}$ package rptR and the rpt.remlLMM procedure to test for the significance of repeatability index with likelihood ratio tests and to estimate repeatability confidence intervals using parametric bootstrapping. To test whether repeatability varied with species mobility, we followed the procedure described in Dingemanse et al. (2012b). For each test, we fitted a LMM on the data pooled over the four species (and thus three mobility ranks) and estimated individual and residual variance components for species of each mobility rank simultaneously, which we compared with a reduced model where individual variances of the species with each mobility rank were constrained to the same value. For this purpose, we standardized mobility scores across mobility ranks by rescaling the total variance within mobility rank to one, thereby ensuring that we tested for differences in repeatability rather than individual variance across species with different mobility ranks. Models with different random structures were compared using the AIC, as advised by Zuur et al. (2009).

\section{Correlations between mobility-related traits}

We then tested for correlations between individual mobility scores across tests using linear models with the score at one test (or the mean score for tests with repeated measures) as response variable, the score at another test as an explanatory variable as well as species expected mobility rank, age and sex as fixed variables. As we were primarily interested in testing whether the relationship between scores at two different tests depended on species mobility rank, we tested the interaction between test score and species mobility rank. Age was never significant and was excluded from the models. We also considered potential effects of morphology (i.e. wing loading and aspect-ratio) on the correlations between traits, but it had no qualitative effect on the results (see Supplementary material Appendix 1 for details on morphology measurements and models including morphology).

\section{Results}

\section{Among-species differences in mobility-related traits}

Mobility assessed with the vortex test was significantly different among species mobility rank (Kruskall-Wallis $\chi^{2}=0.52$; $\mathrm{DF}=2 ; \mathrm{p}=0.008)$, whereas the mobility rank effect was marginally significant for the tunnel test $(p=0.078)$, and not significant for the greenhouse test $(p=0.863)$. Heating rate did not differ among species with different mobility ranks $(p=0.210)$ (Table 1, Fig. 2). Species ranking differed among mobility-related traits (Fig. 2, Supplementary material Appendix 2). According to the vortex test, P. rapae was the most mobile species, differing significantly from P. tithonus $(\mathrm{p}=0.022)$ and $M$. jurtina $(\mathrm{p}=0.001)$.

Table 1. Models testing for interspecific differences in greenhouse, tunnel and heating rate scores. Females were taken as reference. Differences in vortex scores are not presented here as vortex flight time was not normally distributed (see text and Fig. 2 for details). Significant effects are in bold.

\begin{tabular}{lcrrr}
\hline Response variable & Explanatory variables & Estimate \pm SE & $t$ & \multicolumn{1}{c}{$\mathrm{t}$} \\
\hline Tunnel distance & mobility rank & $0.110 \pm 0.062$ & 1.766 & 0.078 \\
& sex & $0.135 \pm 0.086$ & 1.562 & 0.119 \\
Greenhouse flight distance & mobility rank & $0.015 \pm 0.086$ & 0.172 & 0.863 \\
& sex & $\mathbf{0 . 6 1 2} \pm \mathbf{0 . 0 9 4}$ & $\mathbf{6 . 5 1}$ & -1.256 \\
Heating rate & mobility rank & $-0.080 \pm 0.064$ & $\mathbf{0 . 0 0 1}$ \\
& sex & $\mathbf{0 . 2 7 3} \pm \mathbf{0 . 0 8 4}$ & $\mathbf{3 . 2 6 7}$ & $\mathbf{0 . 0 0 1}$ \\
\hline
\end{tabular}


Moreover, $M$. jurtina which obtained the lowest score in the vortex test was significantly different from $P$. aegeria $(p=0.005)$. Other between-species differences were not significant $(p>0.1)$. Strong between-sex differences that were consistent across species were also found for three of the four mobility-related traits, males being significantly more mobile than females. Males flew further in the greenhouse $(\mathrm{p}<0.001 ;$ mean flight distance in males $=11.02 \pm 0.76 \mathrm{~m}$; in females $=4.26 \pm 0.44 \mathrm{~m}$; Fig. 2) and warmed up faster $(\mathrm{p}=0.002$; mean heating rate in males $=0.12 \pm 0.06$; in females $=-0.16 \pm 0.05$; Fig. 2). Males also tended to fly longer in the vortex (except for P. rapae and M. jurtina where the difference was not significant) (Fig. 2, Supplementary material Appendix 3).

\section{Individual repeatability of mobility score across time}

Whenever we could test for individual repeatability of mobility score, i.e. for greenhouse flight distance and tunnel distance, we found strong support for it for each mobilityrelated trait within each species (Table 2). We only found null values of repeatability in $P$. tithonus. The models allowing each mobility rank to show different intra-individual variation did not fit the data better than those where intra-individual variation was constrained to the same value (the AIC value was greater when allowing different mobility ranks to show different intra-individual variation: $\triangle \mathrm{AIC}=5.3$ for greenhouse flight distance, $\triangle \mathrm{AIC}=0.50$ for tunnel distance).

\section{Correlations between mobility-related traits}

Most mobility-related traits were inter-correlated, except heating rate that we found independent from any of the three other mobility-related traits (Table 3). Individuals flying over longer distances in the greenhouse flew during a longer time in the vortex (estimate $=0.159 \pm 0.067 ; \mathrm{p}=0.019$ ), independently of species mobility rank. In contrast, the two correlations involving tunnel distance as a response variable depended on the interaction between mobility rank and respectively, greenhouse flight distance and vortex flight time. To disentangle these interaction effects, we used linear models and tested the correlation between tunnel score and greenhouse score (resp. vortex score) within each species. Sex and its interaction with greenhouse score (resp. vortex score) were added in the models to account for potential sexdependent correlations, but they were never significant and were further excluded from the models. Tunnel distance and greenhouse flight distance were significantly correlated in $P$ rapae only (estimate $=0.257 \pm 0.091, \quad \mathrm{p}=0.006$; $\mathrm{p}>0.200$ in the three other species), $P$. rapae butterflies travelling further in the tunnel also flying further in the greenhouse (Fig. 3a). The distance travelled in the tunnel was also positively correlated to the flight time in the vortex in $P$. rapae (estimate $=0.017 \pm 0.005, \mathrm{p}=0.001$ ) and $P$. aegeria (estimate $=0.010 \pm 0.003, \mathrm{p}=0.005)$, but not in M. jurtina $(\mathrm{p}=0.429)$ and $P$. tithonus $(\mathrm{p}=0.616)$. Indeed, $P$. rapae and $P$. aegeria flying further in the tunnel also flew over a longer time in the vortex (Fig. 3b).

\section{Discussion}

Studies investigating mobility syndromes by jointly analyzing behavioral and physiological traits are rather rare, except in the rapidly growing field of personality-dependent dispersal. In addition, in the larger context of behavioral syndromes, interspecific comparisons of syndromes often result from reviews and meta-analyses which combine species-specific studies (Réale et al. 2007, Cote et al. 2010). Such comparisons necessarily lead to biases related to differences in the devices used to test the same behavior. Here, we used exactly the same devices in the same conditions to measure different traits related to mobility in four butterfly species in order to limit the possibility of bias arising from the experimental protocol.

\section{Among-species differences in mobility-related traits}

First, we found that species mobility rank explained differences in species' scores at the vortex test measuring flight endurance under stressful conditions, whereas the effect of mobility rank on the scores at the tunnel test measuring flight willingness under stressful and unfamiliar conditions was only marginally significant. Scores at the greenhouse test and heating experiment were not explained by species mobility. Intriguingly, species which showed the highest score at one test did not necessarily had a higher score at another test; for example, $M$. jurtina had the best mean score at the tunnel but the lowest at the vortex. Moreover, the species' mobility ranking in our study did not necessarily match those from the literature and depended upon mobility-related traits. The most striking difference was those observed between species' ranking on heating rate and those on any other traits (note however that mobility rank did not affect heating rate). Indeed, although the most mobile species in the wild $P$. rapae

Table 2. Repeatability and mean values of greenhouse and tunnel scores. $n=$ sample size; $S E=$ standard error.

\begin{tabular}{|c|c|c|c|c|c|c|}
\hline & Species & Mean \pm SE & Repeatability & Repeatability confidence interval & $\mathrm{p}$-value & $\mathrm{n}$ \\
\hline \multirow[t]{4}{*}{ Greenhouse flight distance } & P. tithonus & $1.893 \pm 0.458$ & 0 & {$[0 ; 0.344]$} & 0.458 & 7 \\
\hline & $P$. aegeria & $4.984 \pm 0.422$ & 0.134 & {$[0.040 ; 0.219]$} & $<0.001$ & 120 \\
\hline & M. jurtina & $2.523 \pm 0.408$ & 0.143 & {$[0.002 ; 0.298]$} & 0.005 & 44 \\
\hline & P. rapae & $4.218 \pm 0.450$ & 0.278 & {$[0.182 ; 0.373]$} & $<0.001$ & 116 \\
\hline \multirow[t]{4}{*}{ Tunnel distance } & P. tithonus & $78.474 \pm 6.036$ & 0.110 & {$[0 ; 0.234]$} & 0.138 & 50 \\
\hline & $P$. aegeria & $97.714 \pm 5.183$ & 0.207 & {$[0.126 ; 0.285]$} & $<0.001$ & 120 \\
\hline & M. jurtina & $134.031 \pm 13.321$ & 0.304 & {$[0.141 ; 0.446]$} & $<0.001$ & 47 \\
\hline & P. rapae & $112.726 \pm 6.467$ & 0.362 & {$[0.272 ; 0.444]$} & $<0.001$ & 116 \\
\hline
\end{tabular}


Table 3. Models testing for between-species differences in the relationships between the four tests. Type 3 sum of squares are presented. Significant effects are in bold.

\begin{tabular}{|c|c|c|c|c|c|}
\hline Response variable & Explanatory variables & Sum of squares & DF & $\mathrm{F}$ & $\mathrm{p}$-value \\
\hline \multirow[t]{4}{*}{ Greenhouse flight distance } & vortex flight time & 4.569 & 1 & 8.509 & 0.004 \\
\hline & mobility rank & 0.285 & 1 & 0.5302 & 0.467 \\
\hline & vortex flight time $\times$ mobility rank & 0.649 & 1 & 1.207 & 0.273 \\
\hline & sex & 16.206 & 1 & 30.180 & $<0.001$ \\
\hline \multirow[t]{4}{*}{ Tunnel distance } & greenhouse flight distance & 1.230 & 1 & 2.050 & 0.153 \\
\hline & mobility rank & 0.916 & 1 & 1.526 & 0.218 \\
\hline & greenhouse flight distance $\times$ mobility rank & 2.487 & 1 & 4.145 & 0.043 \\
\hline & sex & 1.035 & 1 & 1.710 & 0.192 \\
\hline \multirow[t]{4}{*}{ Tunnel distance } & vortex flight time & 2.964 & 1 & 5.812 & 0.017 \\
\hline & mobility rank & 6.198 & 1 & 11.922 & $<0.001$ \\
\hline & vortex flight time $\times$ mobility rank & 7.962 & 1 & 15.316 & $<0.001$ \\
\hline & sex & 0.067 & 1 & 0.128 & 0.721 \\
\hline \multirow[t]{4}{*}{ Heating rate } & greenhouse flight distance & 0.032 & 1 & 0.093 & 0.760 \\
\hline & mobility rank & 0.003 & 1 & 0.010 & 0.922 \\
\hline & greenhouse flight distance $\times$ mobility rank & 0.047 & 1 & 0.135 & 0.713 \\
\hline & sex & 3.675 & 1 & 10.545 & 0.001 \\
\hline \multirow[t]{4}{*}{ Heating rate } & tunnel distance & 0.272 & 1 & 0.376 & 0.376 \\
\hline & mobility rank & 0.335 & 1 & 0.326 & 0.326 \\
\hline & tunnel distance $\times$ mobility rank & 0.392 & 1 & 0.288 & 0.288 \\
\hline & $\operatorname{sex}$ & 4.234 & 1 & 12.223 & $<0.001$ \\
\hline \multirow[t]{4}{*}{ Heating rate } & vortex flight time & $<0.001$ & 1 & $<0.001$ & 0.983 \\
\hline & mobility rank & $<0.001$ & 1 & $<0.001$ & 0.993 \\
\hline & vortex flight time $\times$ mobility rank & 0.003 & 1 & 0.010 & 0.922 \\
\hline & Sex & 3.493 & 1 & 9.835 & 0.002 \\
\hline
\end{tabular}

also had higher scores than the less mobile P. tithonus in the wild for the three flight tests (greenhouse, tunnel and vortex), the reverse was true for the heating rate (Fig. 2). Higher heating rate in $P$. tithonus as compared to the other species may be explained by the ability of the species to actively warm up using wing shivering. Indeed, $P$. tithonus individuals were observed shaking their wings during the heating rate measurements, reflecting a strong activity of their thorax muscles likely to increase heating rate. This behavior was not observed in any of the three other species. Finally, our mobility tests did not systematically detect $P$. aegeria and $M$. jurtina as intermediate species regarding their mobility scores, as expected from the literature. Between-species differences in physiology or behavior (such as the ability to cope with stressful conditions in captivity) might explain why species' ranking did not match exactly those observed in the wild, and need to be further investigated.

\section{Individual repeatability of mobility score across time}

Both the distances travelled in the tunnel and in the greenhouse were significantly repeatable in all the species but P. tithonus, although the repeatability estimates were quite low given the short time interval between repeated measurements. These results show that, independently of the species, individuals tended to show consistent scores within each test. The absence of repeatability in $P$. tithonus may be explained by the low number of individuals that we tested, especially in the greenhouse $(n=7)$. Measurements on a larger number of individuals are thus needed to conclude on this species. According to our hypothesis, we expected more mobile species to show stronger within-individual consistency in mobility-related traits across time. We did not find any such pattern, as we did not detect any significant difference in repeatability across time among species, neither in the greenhouse nor in the tunnel. Given the large between-species differences in mean repeatability (for instance, from 0.11 to 0.36 for the tunnel distance), and the large confidence intervals obtained (Table 2), we may however expect that more substantial sample sizes are needed to detect meaningful differences. Note that although not significantly different across species, estimates of repeatability for each test tended to rank according to species' mobility in the wild, the less mobile species ( $P$. tithonus) having the lowest repeatability estimates, and the most mobile species (P. rapae) having the highest estimates. Species showed higher repeatability values in the tunnel test compared to the greenhouse, a pattern consistent with previous results in the large white butterfly Pieris brassicae (Ducatez et al. 2012). This may reflect a larger environmental variance in semi-natural conditions than in the laboratory, increasing intra-individual variation in behavior. It is also noteworthy that $P$. rapae was the species whose repeatability estimates were the closest to repeatability estimates for $P$. brassicae (Ducatez et al. 2012), a species which also has a nomadic strategy of space use and is highly mobile.

\section{Species-specific patterns of correlation between mobility-related traits}

According to our hypothesis, we expected more mobile species to show stronger correlations between traits than less mobile species. Indeed, most correlations between mobility-related traits depended upon species' mobility rank, 

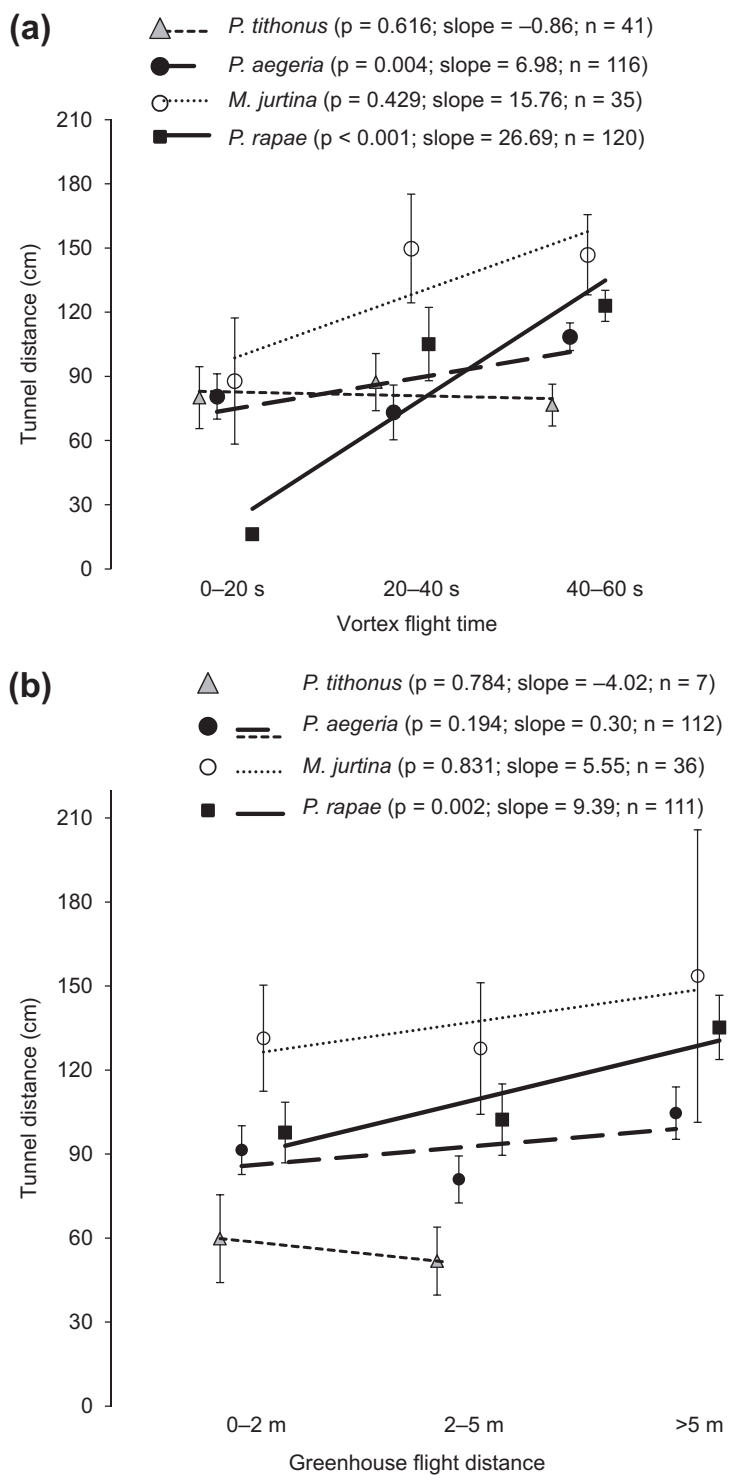

Figure 3. For the four butterfly species, (a) relationship between the distance travelled in the tunnel and the distance travelled in the greenhouse, (b) relationship between the distance travelled in the tunnel and the flight time in the vortex test. The p-values indicate slopes' significance (see text for details). Error bars are standard errors. Note that scores for greenhouse flight distance and vortex flight time were grouped into three categories to avoid overloading the figures, although both variables were considered continuous in the analyses. In P. tithonus, the maximum greenhouse flight distance was of $3.5 \mathrm{~m}$.

more mobile species showing stronger correlations between traits. In the most mobile species $P$. rapae, tunnel distance was significantly and positively correlated with both greenhouse flight distance and vortex flight time. In contrast, these correlations were never significant in the least mobile species $P$. tithonus and the intermediate $M$. jurtina. The absence of correlation in these species might however be explained by the lower statistical power due to lower sample sizes. However, the observation that the slopes of the relationships between mobility related traits increased with species mobility suggest that the correlations were indeed weaker in these species (Fig. 3). Only tunnel distance and vortex flight time correlated significantly (and positively) in the other intermediate species $P$. aegeria. Thus higher mobility in the wild was associated with a stronger syndrome structure in our study, more mobile species having more specialized phenotypes with regards to mobility in our study site.

In our study, we chose to perform behavioral tests with the same temporal sequence of mobility tests. One might expect a negative correlation between scores at successive tests, whenever resources might be limiting. For example, individuals flying longer distance in the greenhouse test might use more energy and could thus perform worse in the vortex test. We did not find any evidence that our results could be explained only by resource allocations. Indeed, we did not find any significant negative correlation between scores in any species, but positive ones for most pairs of tests, making this hypothesis unlikely.

Our results support the hypothesis that mobility syndromes can only be observed for species where different dispersal strategies co-occur (Fig. 1). We indeed expected a given landscape fragmentation level to induce differences in the maintenance of dispersal polymorphism according to species mobility. Our results suggest that in the highly fragmented urban area where we collected the four species, the highly mobile species $P$. rapae still shows a variety of movement strategies, from individuals displaying rather sedentary strategies to nomadic individuals. In contrast, natural selection may have selected for a resident strategy in less mobile species such as $P$. tithonus, dispersal being likely to be too costly for such species in such a highly fragmented landscape. As a result, a mobility syndrome might also exist in less mobile species, although not detectable in such highly fragmented landscapes, where only individuals with the lowest mobility are found, and the between-individual variance in mobility is low. Obviously, the next step to our study would be to measure the syndrome structure of the four species in a larger panel of landscapes (e.g. with varying levels of landscape fragmentation) to better understand the interaction effects of landscape structure and species mobility on the detectability of mobility/dispersal syndromes. In addition, measuring dispersal in the field or in experimental metapopulations (Legrand et al. 2012) would help understanding whether the between-species differences in syndrome structure assessed from mobility-related traits are indeed related to differences in the level of polymorphism of dispersal strategies.

Whether the differences in syndrome structure and consistency across time has a genetic origin, or can change by behavioral flexibility according to the environmental conditions, as found in sticklebacks (Bell and Sih 2007), also remains an important question. However, as the traits measured here in butterflies not only imply behavioral but also physiological characteristics, we may expect a lower flexibility of this syndrome. Genetic and epigenetic factors already known to affect dispersal in butterflies are likely to play an important role in the origin and maintenance of this syndrome (Wheat 2012).

In this study, we interpreted our results according to between-species differences in mobility documented in the wild. However, the four species differ in other traits, including ecological traits likely to affect their responses to landscape structure, and their movement strategies (Andrén et al. 1997). Especially, P. rapae is known as a habitat 
generalist species, inhabiting any type of open habitat, whereas the three other species are more specialized in one type of land cover (e.g. forest or grasslands). Dispersal in urban areas might also be less costly for generalist species, as moving individuals are more likely to frequently encounter new suitable areas. Both a higher mobility and a lower specialization may explain the maintenance of multiple individual strategies in P. rapae compared to less mobile and more specialized species. In addition, although species are treated as fully independent units throughout the study, we could expect phylogenetic relationships to explain parts of the observed patterns. The most closely related species $P$. tithonus and $M$. jurtina show no correlations between traits, whereas the strongest syndrome structure was observed in P. rapae, the most distant species from the others. However, although $P$. aegeria is more closely related to $P$. tithonus and $M$. jurtina (all three species are in the same family) than to P. rapae, it shows an intermediate syndrome structure. In addition, the important differences in mobility test scores between the three most closely related species suggests that phylogeny was not a good predictor of species relative scores at the different tests. This is in accordance with results obtained by Stevens et al. (2012), which show that phylogeny has negligible influence on mobility in European butterflies.

Between individuals differences in condition could also have affected the results, so that poor condition individuals would consistently perform worth than good condition ones. However, we took this potential bias into account, using different proxies of condition as explanatory variables in our analyses and did not find any evidence that individual conditions alone may explain our results. First, we tested for an effect of age using an age index classically used in the literature (Baguette and Nève 1994) based on the wear of the wings and varying from 1 [fresh] to 4 [extensive wing wear]. We found that it did not affect mobility scores at any of the tests. Second, we ran an analysis including morphological variables (wing morphology and wing loading) likely to be related to individuals' condition, as fixed explanatory variables (Supplementary material Appendix 1 ). None of these variables significantly affected the scores at the different tests, nor the correlations between tests scores. We also measured the number of days individuals survived in the lab after the tests (which varied from 1 to 14 ), and found that it was not related to the mobility score at any of the 4 tests (results not shown). All together, these results suggest that between-individuals differences in condition did not affect our results.

In contrast to a previous study in Pieris brassicae (Ducatez et al. 2012), the patterns of correlation among mobility-related traits did not depend on sex or morphology (see also Supplementary material Appendix 1). In addition, we did not find any relationship between heating rate and the other tests, whereas individuals getting warm faster also had a higher flight time in the vortex in $P$. brassicae in Ducatez (2011). Our experimental measure of heating rate however focused on passive heating capacity (except for the wing shivering species $P$. tithonus, see above). Indeed, contrarily to heating in the wild, individuals could not raise their temperature by basking in sunlight, but could mainly passively reach the ambient temperature. Complementary experiments on heating rates in natural conditions might bring more information on the importance of heating rate and more generally thermoregulation performances in the mobility syndrome.

\section{Between-sex differences within species}

We observed strong differences between sexes in three out of the four tests, males being more mobile than females. Males travelled a longer distance in the greenhouse, flew more time in the vortex and warmed up faster than females. Between-sex differences in selection pressures are likely to explain such patterns. Indeed, males have been shown to have better movement capacities than females in many butterfly species (Karlsson and Wickman 1990, Berwaerts et al. 2002, 2008, Turlure et al. 2010), a proximal explanation being that females are generally heavier than males and allocate more of their energy into abdomen biomass than do males, and thus have relatively fewer flight muscles than males per unit of body mass (Berwaerts et al. 2002).

As illustrated by the absence of interaction effects between species and sex on the tests scores, the effect of sex was similar for the four species, except for the vortex test. The strength of the between-sex differences in mobility thus appears rather consistent across species. This result could seem rather surprising. Indeed, the four species have very different reproductive behaviors associated with mobility. In $P$. aegeria for example, most males are territorial and display a 'perching' strategy, where they wait for females at a particular place (Merckx and Van Dyck 2005). Such a behavior is not observed in the three other species, which are supposed to have a 'patroller' strategy, where males actively search for females. The strength of sexual selection acting on mobility in both types of strategies could have led to strong inter-specific variations in the between-sex differences in mobility, as illustrated by stronger differences between males and females scores at the vortex test in $P$. aegeria than in $M$. jurtina or $P$. rapae. It is however noteworthy that mate locating strategies had no impact on dispersal, as shown by a quantitative analysis of life-history traits in European butterflies (Stevens et al. 2012).

\section{Conclusion}

Overall, our results show differences in mobility related syndromes in four common butterfly species originating from the same locality. Such a pattern raises the question of whether these differences indeed arise from differences in the degree of polymorphism in dispersal strategy in the sampled locality. Moreover, it raises the question of the proximate mechanisms at the origin of the syndrome. Under an adaptive hypothesis, we can expect that selective pressures on resident/disperser or resident/resource tracking strategies resulted in different traits associations in species with different dispersal costs in fragmented landscapes. A way to test this adaptive hypothesis would be to compare the syndrome structure of populations of these species collected in landscapes differing in their geometry. Landscape fragmentation has already been shown to influence mobility in different species (Van Dyck and Matthysen 1999, Merckx et al. 2006, 
Schtikzelle et al. 2006, Dover and Settele 2009), including $P$. brassicae (Ducatez et al. 2013) and P. aegeria (Merckx et al. 2006, Bergerot et al. 2012). We could thus expect withinspecies changes in the syndrome structure according to selective pressures associated with contrasted environmental conditions, as found in fish and birds (Bell and Sih 2007, Dingemanse et al. 2007, 2012a, b, Nicolaus et al. 2012).

Acknowledgements - We thank Y. Ben Chehida, A. Daviot, M. Delavelle, M. Le Gall and A. Raveneau for help in the experimental work and Jean-François Legalliard for suggestions that greatly improved the manuscript. This work was supported by grants from the IFR 101 (Inst. d'Ecologie: Biodiversité, Ecologie, Environnement), the ANR open call projects DIAME (Dispersal and Metapopulation) and INDHET (Individual Heterogeneity) to $\mathrm{MB}$, the ANR 6th extinction project MOBIGEN (Mobility genetics and genomic) to $\mathrm{MB}$ and $\mathrm{HF}$, a $\mathrm{PhD}$ grant from the Ministère de l'Enseignement Supérieur et de la Recherche to SD and a post-doctoral fellowship from the Fondation Fyssen to SD. $\mathrm{MB}$ is part of the 'Laboratoire d'Excellence' (LABEX) entitled TULIP (ANR-10-LABX-41).

\section{References}

Andrén, H. et al. 1997. Population response to landscape changes depends on specialization to different landscape elements. - Oikos 80: 193-196.

Baguette, M. and Nève, G. 1994. Adult movements between populations in the specialist butterfly Proclossiana eunomia. - Ecol. Entomol. 19: 1-5.

Baguette, M. et al. 2012. Evolutionary ecology of dispersal in fragmented landscape. - In: Clobert, J. et al. (eds), Dispersal ecology and evolution. Oxford Univ. Press, pp. 381-391.

Bell, A. M. and Sih, A. 2007. Exposure to predation generates personality in threespined sticklebacks (Gasterosteus aculeatus). - Ecol. Lett. 10: 828-834.

Bergerot, B. et al. 2012. Habitat fragmentation impacts mobility in a common and widespread woodland butterfly: do sexes respond differently? - BMC Ecol. 12: 5.

Berwaerts, K. et al. 2002. Does flight morphology relate to flight performance? An experimental test with the butterfly Pararge aegeria. - Funct. Ecol. 16: 484-491.

Berwaerts, K. et al. 2008. Take-off flight performance in the butterfly Pararge aegeria relative to sex and morphology: a quantitative genetic assessment. - Evolution 62: 2525-2533.

Bonte, D. et al. 2012. Costs of dispersal. - Biol. Rev. 87: 290-312.

Charmantier, A. et al. 2011. Heritability of short-scale natal dispersal in a large-scale foraging bird, the wandering albatross. - J. Evol. Biol. 24: 1487-1496.

Cheptou, P. O. et al. 2008. Rapid evolution of seed dispersal in an urban environment in the weed Crepis sancta. - Proc. Natl Acad. Sci. USA 105: 3796-3799.

Cizek, L. et al. 2006. Host plant defences and voltinism in European butterflies. - Ecol. Entomol. 31: 337-344.

Clobert, J. et al. 2009. Informed dispersal, heterogeneity in animal dispersal syndromes and the dynamics of spatially structured populations. - Ecol. Lett. 12: 197-209.

Clobert, J. et al. 2012. Dispersal ecology and evolution. - Oxford Univ. Press.

Cook, L. M. et al. 2001. Butterfly-hostplant fidelity, vagrancy and measuring mobility from distribution maps. - Ecography 24: 497-504.

Cote, J. et al. 2010. Personality-dependent dispersal: characterization, ontogeny and consequences for spatially structured populations. - Phil. Trans. R. Soc. B 365: 4065-4076.
Dennis, R. L. H. and Shreeve, T. G. 1997. Diversity of butterflies on British islands: ecological influences underlying the roles of area, isolation and the size of the faunal source. - Biol. J. Linn. Soc. 60: 257-275.

Dingemanse, N. J. et al. 2003. Natal dispersal and personalities in great tits (Parus major). - Proc. R. Soc. B 270: 741-747.

Dingemanse, N. et al. 2007. Behavioural syndromes differ predictably between 12 populations of three-spined stickleback. - J. Anim. Ecol. 76: 1128-1138.

Dingemanse, N. J. et al. 2009. Individual experience and evolutionary history of predation affect expression of heritable variation in fish personality and morphology. - Proc. R. Soc. B 276: 1285-1293.

Dingemanse, N. J. et al. 2012a. Quantitative genetics of behavioural reaction norms: genetic correlations between personality and behavioural plasticity vary across stickleback populations. - J. Evol. Biol. 25: 485-496.

Dingemanse, N. J. et al. 2012b. Variation in personality and behavioural plasticity across four populations of the great tit Parus major. - J. Anim. Ecol. 81: 116-126.

Doligez, B. et al. 2009. 'Heritability' of dispersal propensity in a patchy population. - Proc. R. Soc. B 276: 2829-2836.

Dover, J. and Settele, J. 2009. The influences of landscape structure on butterfly distribution and movement: a review. - J. Insect Conserv. 13: 3-27.

Ducatez, S. 2011. Evolution of mobility in butterflies: constraints and adaptations. - PhD thesis, Museum National d'Histoire Naturelle, Paris.

Ducatez, S. et al. 2012. Inter-individual variation in movement: is there a mobility syndrome in the large white butterfly Pieris brassicae? - Ecol. Entomol. 37: 377-385.

Ducatez, S. et al. 2013. Flight endurance and heating rate vary with both latitude and habitat connectivity in a butterfly species. - Oikos 122: 601-611.

Haag, C. R. et al. 2005. A candidate locus for variation in dispersal rate in a butterfly metapopulation. - Proc. R. Soc. B 272: 2449-2456.

Hansson, B. et al. 2003. Heritability of dispersal in the great reed warbler. - Ecol. Lett. 6: 290-294.

Karlsson, B. and Wickman, P. O. 1990. Increase in reproductive effort as explained by body size and ressource-allocation in the speckled wood butterfly Pararge aegeria (L). - Funct. Ecol. 4: 609-617.

Lafranchis, T. 2004. Butterflies of Europe. - Diatheo, Paris.

Legrand, D. et al. 2012. The Metatron: an experimental system to study dispersal and metaecosystems for terrestrial organisms. - Nat. Methods 9: 828-833.

Lynch, M. and Walsh, B. 1998. Genetics and analysis of quantitative traits. - Sinauer.

Merckx, T. and Van Dyck, H 2005. Mate location behaviour of the butterfly Pararge aegeria in woodland and fragmented landscapes. - Anim. Behav. 70: 411-416.

Merckx, T. et al. 2006. Sex- and landscape-related differences in flight ability under suboptimal temperatures in a woodland butterfly. - Funct. Ecol. 20: 436-441.

Mueller, T. and Fagan, W. F. 2008. Search and navigation in dynamic environments - from individual behaviors to population distributions. - Oikos 117: 654-664.

Nakagawa, S. and Schielzeth, H. 2010. Repeatability for Gaussian and non-Gaussian data: a practical guide for biologists. - Biol. Rev. 85: 935-956.

Nicolaus, M. et al. 2012. Experimental evidence for adaptive personalities in a wild passerine bird. - Proc. R. Soc. B 279: 4885-4892.

Niitepold, K. et al. 2009. Flight metabolic rate and Pgi genotype influence butterfly dispersal rate in the field. - Ecology 90: 2223-2232. 
Pollard, E. and Yates, T. J. 1993. Monitoring butterflies for ecology and conservation. - Chapman and Hall.

Réale, D. et al. 2007. Integrating animal temperament within ecology and evolution. - Biol. Rev. 82: 291-318.

Ronce, O. 2007. How does it feel to be like a rolling stone? Ten questions about dispersal evolution. - Annu. Rev. Ecol. Evol. Syst. 38: 231-253.

Schtikzelle, N. et al. 2006. Dispersal depression with habitat fragmentation in the bog fritillary butterfly. - Ecology 87: 1057-1065.

Stevens, V. M. et al. 2010a. A meta-analysis of dispersal in butterflies. - Biol. Rev. 85: 625-642.

Stevens, V. M. et al. 2010b. Variation within and between closely related species uncovers high intra-specific variability in dispersal. - PLoS One 5: e11123.

Stevens, V. M. et al. 2012. How is dispersal integrated in life histories: a quantitative analysis using butterflies. - Ecol. Lett. 15: 74-86.

Thomas, J. A. 1984. The conservation of butterflies in temperate countries: past efforts and lessons for the future.

Supplementary material (Appendix ECOG-00365 at < www. oikosoffice.lu.se/appendix $>$ ). Appendix 1-3.
- In: Vane-Wright, R. I. and Ackery, P. R. (eds), The biology of butterflies. Academic Press, pp. 333-353.

Turlure, C. et al. 2010. Resource grain scales mobility and adult morphology in butterflies. - Landscape Ecol. 25: 95-108.

Van Dyck, H. and Matthysen, E. 1998. Thermoregulatory differences between phenotypes in the speckled wood butterfly: hot perchers and cold patrollers? - Oecologia 114: 326-334.

Van Dyck, H. and Matthysen, E. 1999. Habitat fragmentation and insect flight: a changing 'design' in a changing landscape? - Trends Ecol. Evol. 14: 172-174.

Van Dyck, H. and Baguette, M. 2012. Dispersal and fragmentation in invertebrates - examples from widespread and localized butterflies. - In: Clobert, J. et al. (eds), Dispersal ecology and evolution. Oxford Univ. Press, pp. 420-429.

Wheat, C. W. 2012. Dispersal genetics: emerging insights from fruitflies, butterflies, and beyond. - In: Clobert, J. et al. (eds), Dispersal ecology and evolution. Oxford Univ. Press, pp. 95-107.

Zuur, A. F. et al. 2009. Mixed effects models and extensions in ecology with R. - Springer. 